\title{
Graphene oxide waveguide and micro-ring resonator polarizers
}

\author{
Yang $\mathrm{Qu}^{\mathrm{a}}$, Jiayang $\mathrm{Wu}^{\mathrm{a}}$, Yunyi Yang ${ }^{\mathrm{a}}$, Xingyuan $\mathrm{Xu}^{\mathrm{a}}$, Yao Liang ${ }^{\mathrm{a}}$, Sai T. Chu ${ }^{\mathrm{b}}$, Brent E. Little \\ Roberto Morandotti ${ }^{\mathrm{de}, \mathrm{f}}$, Baohua Jia, ${ }^{\mathrm{a}, *}$ and David J. Moss ${ }^{\mathrm{a}, *}$ \\ ${ }^{a}$ Centre for Micro-Photonics, Swinburne University of Technology, Hawthorn, VIC 3122, Australia \\ ${ }^{\mathrm{b}}$ City University of Hong Kong, Tat Chee Avenue, Hong Kong, China \\ 'Xi'an Institute of Optics and Precision Mechanics Precision Mechanics of CAS, Xi'an, China \\ ${ }^{d}$ INRS -Énergie, Matériaux et Télécommunications, 1650 Boulevard Lionel-Boulet, Varennes, Québec, Canada \\ 'ITMO University, St. Petersburg, Russia \\ ${ }^{f}$ University of Electronic Science and Technology of China, Chengdu 610054, China. \\ *E-mail: bjia@swin.edu.au, dmoss@ swin.edu.au
}

\begin{abstract}
We experimentally demonstrate integrated waveguide polarizers and polarization-selective micro-ring resonators (MRRs) incorporated with layered graphene oxide (GO) films. We achieve a high polarization dependent loss (PDL) of $\sim 53.8 \mathrm{~dB}$ for the GO-coated waveguide and a high polarization extinction ratio of $\sim 8.3 \mathrm{~dB}$ for the GO-coated MRR.
\end{abstract}

Keywords: 2D materials, polarizer, integrated photonics, material anisotropy, graphene oxide

\section{INTRODUCTION}

Polarization control is a fundamental requirement in many optical technologies [1,2]. Integrated polarization-selective devices based on complementary metal-oxide-semiconductor (CMOS) compatible integrated platforms [3], offering advantages of compact footprint, high stability, mass producibility and high scalability, are functional building blocks for photonic integrated circuits [2]. In recent years, the huge optical anisotropy and broadband response of two-dimensional (2D) materials such as graphene and transition metal dichalcogenides have been widely recognized and exploited to implement polarization-selective devices [4-7]. Owing to its ease of preparation as well as the tunability of its material properties, graphene oxide (GO) has become a highly promising member of the 2D family [8,9]. A broadband GO-polymer waveguide polarizer with a high PDL of $\sim 40 \mathrm{~dB}$ was demonstrated, where the GO films were introduced onto an SU8 polymer waveguide using drop-casting methods. The GO film thickness for each drop-casting step was $\sim 0.5 \mu \mathrm{m}$ and the drop coating diameter was $\sim 1.3 \mathrm{~mm}$, neither being ideal for achieving precise control of the placement, thickness, and length of the GO films.

Recently [10-16], we reported large-area, transfer-free, and high-quality GO film coating on integrated waveguides using a solution-based method with layer-by-layer deposition of GO films. Here, we use these techniques to demonstrate GO-coated integrated waveguide polarizers and polarization-selective micro-ring resonators (MRRs) on a CMOS compatible doped silica platform. We achieve highly precise control of the placement, thickness, and length of the GO films coated on integrated photonic devices by using our layer-by-layer GO coating method followed by photolithography and lift-off processes. We measure the performance of the waveguide polarizer for different GO film thicknesses and lengths versus polarization, wavelength, and power, achieving a very high polarization dependent loss (PDL) of $\sim 53.8 \mathrm{~dB}$. For GO-coated integrated MRRs, we achieve an $8.3-\mathrm{dB}$ polarization extinction ratio between the TE and TM resonances. These results confirm the high-performance of integrated polarization selective devices incorporated with GO films.

\section{DEVICE FABRICATION AND EXEPERIMENTAL RESULTS}

The integrated waveguides were fabricated from high-index doped silica glass core $(\sim 2 \mu \mathrm{m} \times \sim 1.5 \mu \mathrm{m})$ surrounded by silica via CMOS compatible process, with chemical mechanical polishing used to remove the upper cladding to enable GO film coating on the top surface of the waveguide. GO coating was achieved with a solution-based method that yielded layer-by-layer GO film deposition, as reported previously [10,11]. Our solution-based GO coating approach, unlike for example, the sophisticated transfer processes employed for coating 2D materials such as graphene [12], is capable of coating large areas (e.g., a 4 inch wafer) on dielectric substrates (e.g., silicon and silica wafers) with relatively few defects. Figure 1 (a) shows the measured Raman spectra of the waveguides without GO and with 2 layers of GO. Figure 1(b) shows

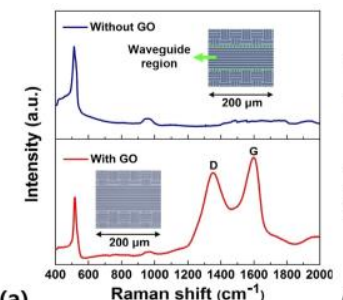

(a)

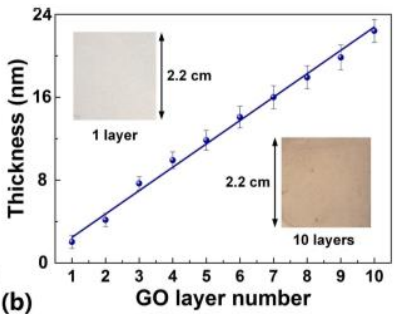

(b)

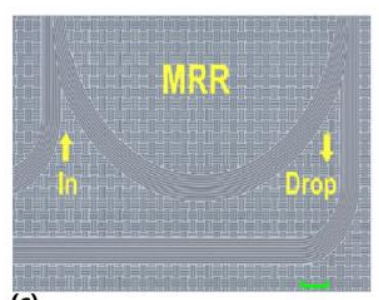

(c)

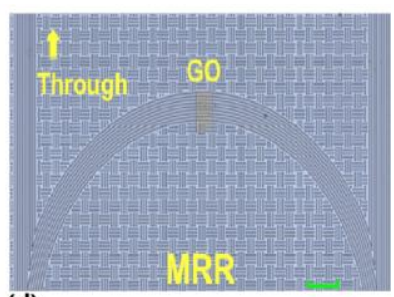

(d)

Figure 1. (a) Raman spectra of integrated chip without GO and with 2 layers of GO. Insets show the corresponding microscope images. (b) Measured GO film thickness versus GO layer number. Insets show the images of a silica substrate coated with 1 and 10 layers of GO. (c)-(d) Microscope images of the MRR uniformly coated with 5 layers of GO and patterned with 50 layers of GO, respectively. 

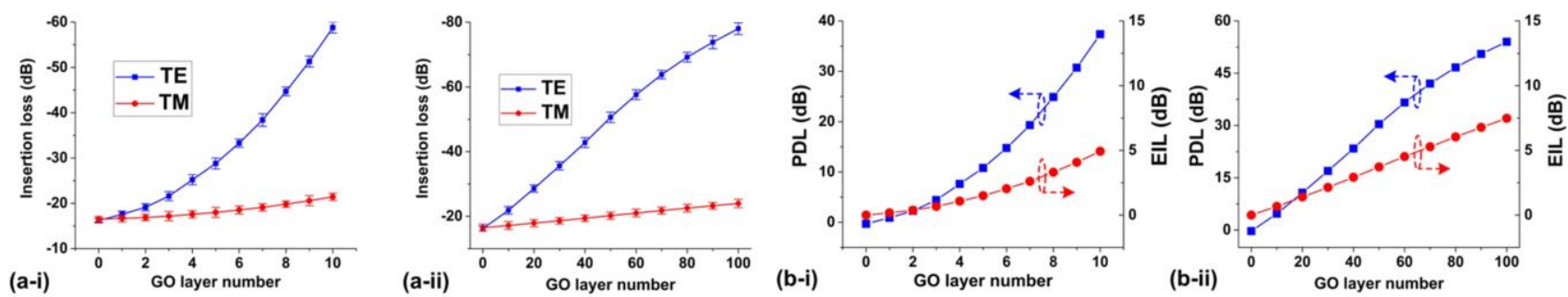

Figure 2 (a) Measured TE and TM polarized insertion loss. (b) Extracted PDL and EIL. In (a) and (b), (i) shows the results for 1.5-cmlong uniformly coated waveguides $(0,1,2, \ldots, 10$ layers of GO) and (ii) shows the results for 1.5 -cm-long waveguides with 2-mm-long patterned $\mathrm{GO}(0,10,20, \ldots, 100$ layers of $\mathrm{GO})$. The input $\mathrm{CW}$ power and wavelength in (a) are $0 \mathrm{dBm}$ and $1550 \mathrm{~nm}$.

the thickness of GO films versus the layer number characterized by atomic force microscopy. In addition to the uniformly coated devices, we selectively patterned areas of GO films using lithography and lift-off processes, which allows precise control of the size and placement of the GO films, Figure 1(c) and (d) show microscope images of the integrated MRR uniformly coated with 5 layers of GO and patterned with 50 layers of GO, respectively. Note that although a number of concentric rings are shown, only the center ring was coupled with the through/drop bus waveguides to form a MRR.

Figure 2 shows the polarization dependent performance for both the $1.5-\mathrm{cm}$-long uniformly coated waveguides $(0-10$ layers, (i)) and the patterned 2-mm-long devices (10-100 layers, (ii)). The TE insertion loss increases much more strongly than TM with layer number, thus yielding a large PDL with low EIL (defined as the insertion loss induced by the GO film over the uncoated waveguide). The PDL reached a maximum of $\sim 37.4 \mathrm{~dB}$ for a 10 layer uniformly coated device and $\sim 53.8$ $\mathrm{dB}$ for a 100 layer patterned device, with a modest maximum EIL of $\sim 5.0 \mathrm{~dB}$ and $\sim 7.5 \mathrm{~dB}$ for the two devices, respectively.

The measured TE and TM polarized transmission spectra of the uniformly GO-coated MRR are shown in Fig. 3(a), while the transmission spectra of the patterned ( $50 \mu \mathrm{m}$ in length) MRR are shown in Fig. 3(b). For the patterned MRR with 50 layers of GO, a maximum polarization extinction ratio (defined as the difference between the extinction ratios of the TE and TM polarized resonances) of $\sim 8.3 \mathrm{~dB}$ was achieved. These devices would be useful for silicon based photonic chips $[17,18]$, and this concept would also be applicable to other 2D materials as well [19, 20].

(a-i) TE

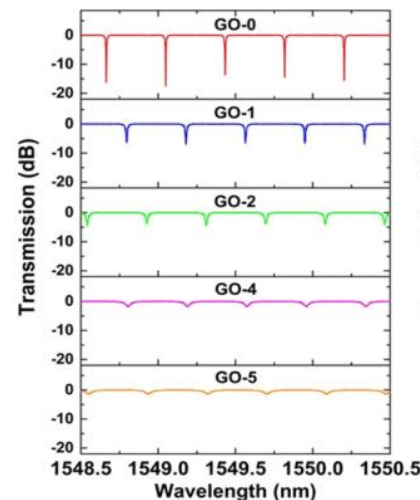

(b-i) TE

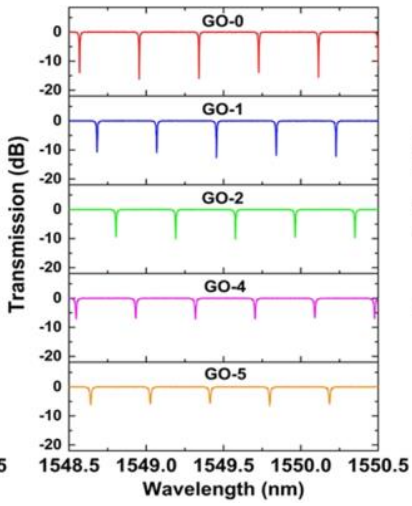

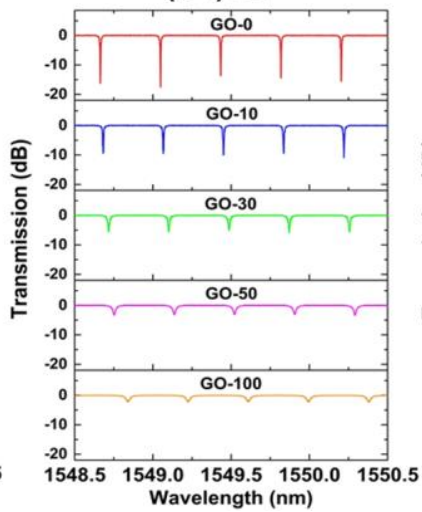

\section{REFERENCES}

[1] Y. Yan et al., "High-capacity millimetre-wave communications with orbital angular momentum multiplexing," Nat. Commun., 5, 4876 (2014).

[2] D. Dai et al., "Passive technologies for future large-scale photonic integrated circuits on silicon: polarization handling, light nonreciprocity and loss reduction," Light Sci. Appl., 1: e1 (2012).

[3] D. Moss et al., "New CMOS-compatible platforms based on silicon nitride and Hydex for nonlinear optics," Nat. Photon., vol. 7, 597 (2013).

[4] Q. Bao et al., "Broadband graphene polarizer," Nat. Photon., 5, 411 (2011).

[5] W. Lim et al., "Graphene oxide-based waveguide polariser: from thin film to quasi-bulk," Opt. Exp., 22 (9), 11090-11098 (2014).

[6] L. Lin et al., "Chalcogenide glass-on-graphene photonics" Nat. Photonics, 11, 798 (2017).

[7] S. Sathiyan et al., "Evolution of the polarizing effect of $\mathrm{MoS}_{2}$ ", IEEE Photon. J., 7 (2015).

[8] K. P. Loh et al., "Graphene oxide as a chemically tunable platform for optical applications", Nat. Chem., 2, 1015 (2010).

[9] X. Zheng et al., "In ditu third-order non-linear responses during laser reduction of graphene oxide thin films towards on-chip nonlinear photonic devices", Adv. Mater., 26, 2699 (2014).

[10] Y. Yang et al., "Enhanced four-wave mixing in waveguides integrated with graphene oxide", APL Photonics, $\underline{\mathbf{3}}$, 120803 (2018).

[11] Y. Yang, et al., "Graphene-Based Multilayered Metamaterials with Phototunable Architecture for On-Chip Photonic Devices", ACS Photonics, 6, 1033 (2019). 
[12] Yang, Y. et al., "Bottom-up Fabrication of Graphene on Silicon/Silica Substrate via a Facile Soft-hard Template Approach", Sci Rep, 5: p. 13480. (2015).

[13] Yang Qu, Jiayang Wu, Yunyi Yang, Yuning Zhang, Corrado Sciancalepore, Christian Grillet, Christelle Monat, Baohua Jia, and David J. Moss, "Enhanced nonlinear four-wave mixing in silicon nitride waveguides integrated with 2D layered graphene oxide films", Advanced Optical Materials $\underline{8}$ (20) 2001048 (2020). DOI: 10.1002/adom.202001048 (2020).

[14] Yuning Zhang, Yang Qu, Jiayang Wu, Linnan Jia, Yunyi Yang, Xingyuan Xu, Baohua Jia, and David J. Moss, "Enhanced Kerr nonlinearity and nonlinear figure of merit in silicon nanowires integrated with 2D graphene oxide films", ACS Applied Materials and Interfaces $\underline{12}$ (29) 33094-33103 June 29 (2020). DOI:10.1021/acsami.0c07852

[15] Jiayang Wu, Yunyi Yang, Yang Qu, Yuning Zhang, Linnan Jia, Xingyuan Xu, Sai T. Chu, Brent E. Little, Roberto Morandotti, Baohua $\mathrm{Jia}$, and David J. Moss, "Enhanced nonlinear four-wave mixing in microring resonators integrated with layered graphene oxide films", Small 16 (16) 1906563 April 23 (2020). DOI: 10.1002/smll.20190656

[16] Jiayang Wu, Yunyi Yang, Yang Qu, Xingyuan Xu, Yao Liang, Sai T. Chu, Brent E. Little, Roberto Morandotti, Baohua Jia, and David J. Moss, "Graphene oxide waveguide polarizers and polarization selective micro-ring resonators", Laser and Photonics Reviews 13 (9) 1900056 (2019). DOI:10.1002/lpor.201900056.

[17] Jiayang Wu, Tania Moein, Xingyuan Xu, and David J. Moss, "Advanced photonic filters via cascaded Sagnac loop reflector resonators in silicon-on-insulator integrated nanowires", Applied Physics Letters Photonics, Vol. 3, 046102 (2018). DOI:/10.1063/1.5025833

[18] Jiayang Wu, Tania Moein, Xingyuan Xu, Guanghui Ren, Arnan Mitchell, and David J. Moss, "Micro-ring resonator quality factor enhancement via an integrated Fabry-Perot cavity", Applied Physics Letters Photonics, vol. 2, 056103 (2017);

doi: $10.1063 / 1.4981392$.

[19] Linnan Jia, Dandan Cui, Jiayang Wu, Haifeng Feng, Tieshan Yang, Yunyi Yang, Yi Du, Weichang Hao, Baohua Jia, David J. Moss, "BiOBr nanoflakes with strong nonlinear optical properties towards hybrid integrated photonic devices", Applied Physics Letters Photonics, vol. 4, 090802 (2019). DOI: 10.1063/1.5116621

[20] Linnan Jia, Jiayang Wu, Yunyi Yang, Yi Du, Baohua Jia, David J. Moss, “Large Third-Order Optical Kerr Nonlinearity in NanometerThick PdSe2 2D Dichalcogenide Films: Implications for Nonlinear Photonic Devices”, ACS Applied Nano Materials, vol. 3, no. 7, 6876-6883 (2020). DOI:10.1021/acsanm.0c01239. 